\title{
Suction deglutition to swallowing deglutition by cortical or subcortical networks
}

\author{
Patrick Fellus* \\ Président of the French Pediatric Orthodontic Society, University Hospital Robert Debré Paris, France
}

\section{Submit}

Dropping the suction deglutition is necessary to build a physiological occlusion. Generally, it occurs by itself for $60 \%$ of children when they are four years old; it is in this sample of children that we find the ones who never need any orthodontic treatment.

When a child requires one, acquiring a good swallowing is going to reduce the duration of the treatment but also ensure the stability of the results and avoid the risk of relapse. This change of the swallowing program can be either done by the cortical or the subcortical way, but the expected results would be different.

As an orthodontists, when we have to fix deformities they are mostly caused by the lack of balance between the opposing forces produced by the cheek muscles and the forces generated by the tongue. Whether orthopaedic treatment in deciduous dentition, orthodontic treatment in the mixed or permanent dentition, it is mandatory to reach at the end of the treatment a functional balance that will ensure the stability of the results.

Suction deglutition is a physiological function during the first years. It has a paleocortical origin, and the facial nerves control it. But it becomes archaic when the full set of teeth is in place, and when mastication appears. If suction-swallowing habit continues, the forces exerted by the labio-jugal muscles will disturb physiological growth and will lead to various skeletal disorders.

A new swallowing program, controlled by the trigeminal nerve is therefore required. It appears spontaneously by a subcortical action around four years old for $60 \%$ of children by using a pre-existing neurological wiring. It will be among those children that we can find the $50 \%$ of them who will never need any orthodontic treatment. If it does not happen by itself, then, like in a computer, a new program will have to be downloaded.

It is what the practitioner or the speech therapist is going to do through series of exercises to code a new praxis. But even with an accurate cooperation of the child, it is long and complicated.

"Memory is based (...) on the nature of the connection between neurons and the way they use the sensorial information they receive. "

According to Eric Kandel, Nobel Price of Medicine in 2000, when this action comes from the cortical area we have a stimulation of neurotransmitters in our synapses, and when it comes from the subcortical area we have a creation of new synapses. The swallowing rehabilitation cannot be based on the control of voluntary movements but on the stimulation of the subcortical area.

Froggymouth is a new appliance which helps young children from
3 - 4 years old to use the best swallowing method for a toothed patient through the subcortical way and not anymore the suction deglutition method (Figure 1).

This Appliance is based on an idea without any connection between the lips and the tongue, which is the exact definition of suction deglutition.

Froggymouth is not placed inside the mouth but between the lips. It will prevent the upper one from touching the lower one, thus, it avoids the negative pressure that was applied inside the mouth thanks to a water-tight joint around the lips. Indeed, it will force the child to find by himself a new way for the deglutition in the lower part of his brain by raising the upper back of the tongue to the palatal bone.

This action will lead to a eumorphic development of the dental arch.

This appliance has to be worn 15 minutes per day and should not be worn during the night like «trainers ». Indeed, the quality of the

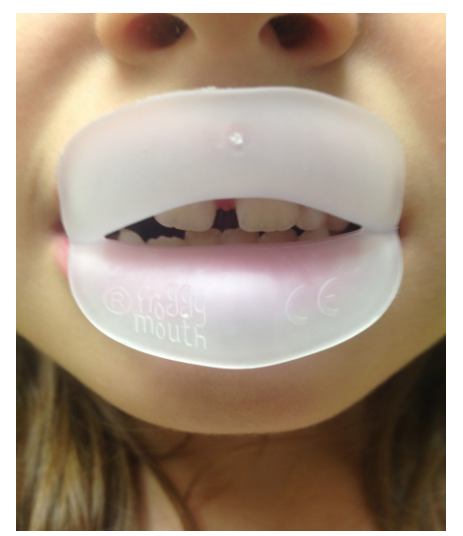

It is based on the principle of the absence of connection between the lips and the tongue, which is the exact definition of suction deglutition.

- This appliance is not placed inside the mouth but betweenthe lips to prevent the upper lip from touching the lower one. Thus it stops the negative pressure to be applied inside the mouth, thanks to a water-tight joint around the lips.

- This will force the child to spontaneously find a new way for deglutition,

Figure 1. Three years old girl wearing Froggy mouth.

Correspondence to: Patrick Fellus, Président of the French Pediatric Orthodontic Society, University Hospital Robert Debré Paris, France, E-mail: fellusp@wanadoo.fr

Received: May 10, 2016; Accepted: June 11, 2016; Published: June 14, 2016 
sleep is critical for young children. It must be worn in front of a screen (TV or movie/series in a computer screen) to catch the attention of the patient fully and then stimulate his neuron circuits which are related to automatic functions.

This Appliance is only an auxiliary that you will be able to use in your treatments whatever the techniques you apply (functional or mechanical) during few weeks. It is obvious that results will be achieved much faster if the muscles and the appliance are both working in the same way rather than if they are fighting against each other.

At rest the lips are not in contact, the teeth are in occlusion, the trigeminal nerve is the leader (Figure 2). But the old swallowing program is used if the lips are in contact the teeth are not closed the facial nerve is the leader a chils swallow with the suction deglutition program (Figure 3).

It will not only reduce the length of your treatments but much more important; it will ensure its durability when the treatment will be over and then reduce the risk of relapse (Figure 4).

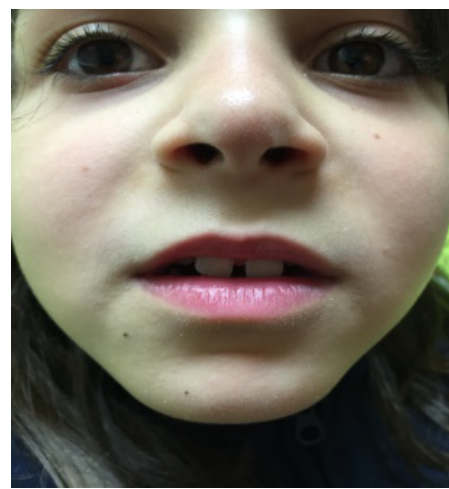

Figure 2: Result after 2 weeks: At rest the lips are not in contact, the teeth are in occlusion, the trigeminal nerve is the leader if the new swallowing program is used.

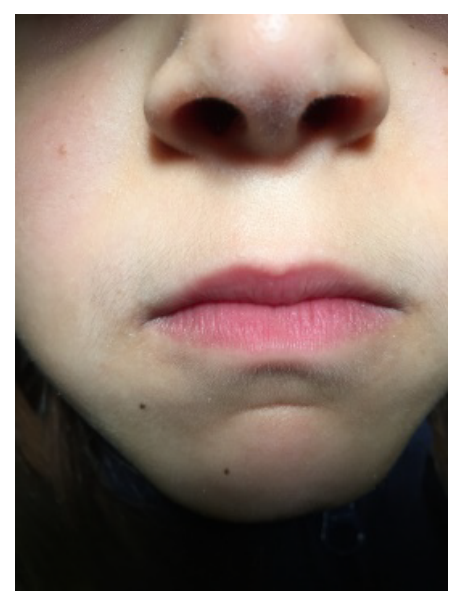

Figure 3. If the lips are in contact the teeth are not closed the facial nerve is the leader the chils swallow with the suction deglutition program.

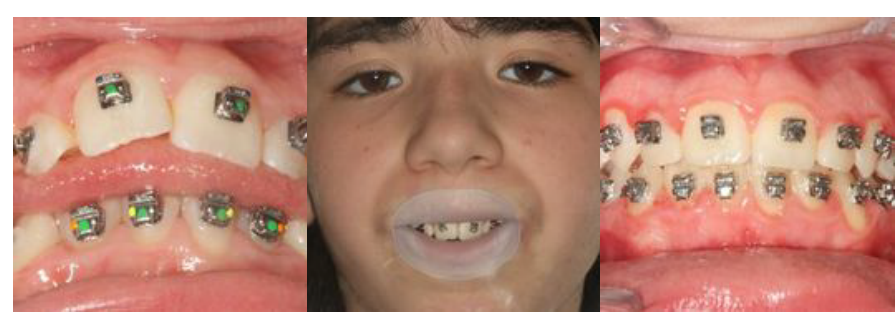

Figure 4. Result of 15 Mins per day with a froggy mouth.

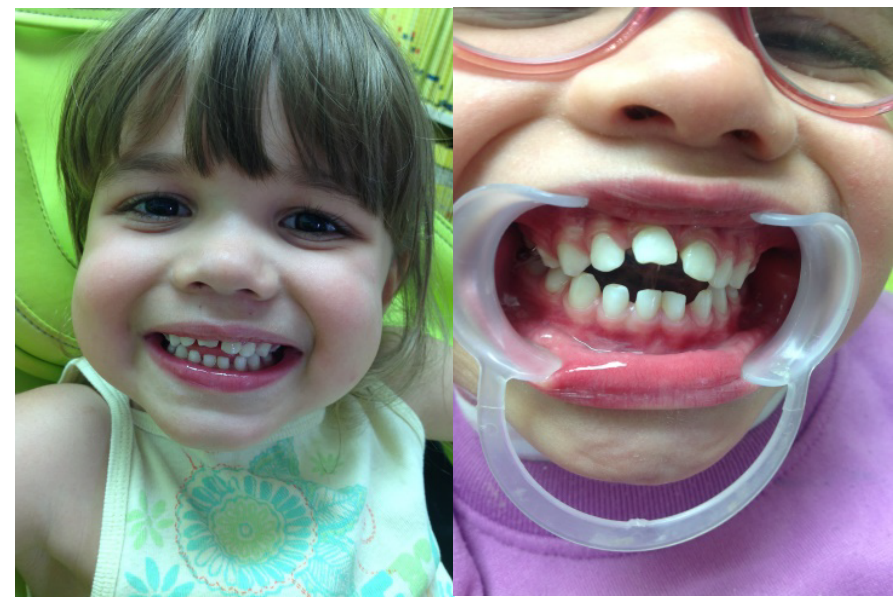

Figure 5. Result 15Min per day with froggy mouth.

Froggy mouth is perfectly adapted to young children and it can even be used alone if the deformity is slight: the simple fact to rebalance the functions will allow a spontaneous correction of slight deformity when the child still has deciduous teeth (Figure 5).

- This Appliance is based on the principle of the absence of connection between the lips and the tongue, which is the exact definition of suction deglutition.

- This appliance is not placed inside the mouth but between the lips to prevent the upper lip from touching the lower one. Thus it stops the negative pressure to be applied inside the mouth, thanks to a water-tight joint around the lips. deglutition.

- This will force the child to spontaneously find a new way for

This little girl of three years old had been wearing Froggy Mouth for only two weeks. She is perfectly at ease with it. She is not dribbling, her face and muscles are relaxed, and the deformity is less significant than the beginning.

Copyright: (C2016 Fellus P. This is an open-access article distributed under the terms of the Creative Commons Attribution License, which permits unrestricted use, distribution, and reproduction in any medium, provided the original author and source are credited. 